\title{
Cochrane
Library
}

Cochrane Database of Systematic Reviews

\section{The champion for improved delivery of care to older people in long-term care settings: effects on professional practice, quality of care and resident outcomes (Protocol)}

Welsh SM, Sherriff A, Flodgren G

Welsh SM, Sherriff A, Flodgren G.

The champion for improved delivery of care to older people in long-term care settings: effects on professional practice, quality of care and resident outcomes.

Cochrane Database of Systematic Reviews 2015, Issue 11. Art. No.: CD011956.

DOI: 10.1002/14651858.CD011956.

www.cochranelibrary.com

The champion for improved delivery of care to older people in long-term care settings: effects on professional practice, quality of care and resident outcomes (Protocol)

Copyright $\odot 2015$ The Cochrane Collaboration. Published by John Wiley \& Sons, Ltd. 
TABLE OF CONTENTS

HEADER . . . . . . . . . . . . . . . . . . . . . . . . . . . . . . . . . . . . 1

ABSTRACT . . . . . . . . . . . . . . . . . . . . . . . . . . . . . . . . . . . . . . . . . .

BACKGROUND . . . . . . . . . . . . . . . . . . . . . . . . . . . . . . . . . . . . . .

OBJECTIVES . . . . . . . . . . . . . . . . . . . . . . . . . . . . . . . . . . . . . . .

METHODS . . . . . . . . . . . . . . . . . . . . . . . . . . . . . . . . . . . . . .

ACKNOWLEDGEMENTS . . . . . . . . . . . . . . . . . . . . . . . . . . . . . . . . . . . . . . . .

REFERENCES . . . . . . . . . . . . . . . . . . . . . . . . . . . . . . . . . . . . . . 6

APPENDICES . . . . . . . . . . . . . . . . . . . . . . . . . . . . . . . . . . . . . 9

CONTRIBUTIONS OF AUTHORS . . . . . . . . . . . . . . . . . . . . . . . . . . . . . . . . . . . . . . . . . . .

DECLARATIONS OF INTEREST . . . . . . . . . . . . . . . . . . . . . . . . . . . . . . . . . 11

SOURCES OF SUPPORT . . . . . . . . . . . . . . . . . . . . . . . . . . . . . . . . . . . . . . . .

The champion for improved delivery of care to older people in long-term care settings: effects on professional practice, quality of care and resident outcomes (Protocol)

Copyright $\odot 2015$ The Cochrane Collaboration. Published by John Wiley \& Sons, Ltd. 


\title{
The champion for improved delivery of care to older people in long-term care settings: effects on professional practice, quality of care and resident outcomes
}

\author{
Sheila M Welsh ${ }^{1}$, Andrea Sherriff ${ }^{2}$, Gerd Flodgren ${ }^{3}$ \\ ${ }^{1}$ Public Health Department, NHS Ayrshire \& Arran, Ayr, UK. ${ }^{2}$ Community Oral Health Section, University of Glasgow Dental \\ School, Glasgow, UK. ${ }^{3}$ Nuffield Department of Population Health, University of Oxford, Oxford, UK \\ Contact address: Sheila M Welsh, Public Health Department, NHS Ayrshire \& Arran, Afton House, Ailsa Campus, Dalmellington \\ Road, Ayr, Ayrshire, KA6 6AB, UK. sheila.welsh@aapct.scot.nhs.uk.
}

Editorial group: Cochrane Effective Practice and Organisation of Care Group.

Publication status and date: New, published in Issue 11, 2015.

Citation: Welsh SM, Sherriff A, Flodgren G. The champion for improved delivery of care to older people in long-term care settings: effects on professional practice, quality of care and resident outcomes. Cochrane Database of Systematic Reviews 2015, Issue 11. Art. No.: CD011956. DOI: 10.1002/14651858.CD011956.

Copyright (C) 2015 The Cochrane Collaboration. Published by John Wiley \& Sons, Ltd.

\begin{abstract}
A B S T R A C T
This is the protocol for a review and there is no abstract. The objectives are as follows:

To assess the effectiveness of the champion on professional practice, quality of care and resident outcomes in long-term care for older people.
\end{abstract}

\section{B A C K G R O U N D}

\section{Description of the condition}

How to deliver high-quality, affordable long-term care for the emerging population of older people who require it has become one of the defining challenges for policy makers and service providers worldwide.

In some developed countries (e.g. Scotland, Australia, Sweden and Denmark), government policy supports efforts towards 'ageing in place', which enable older people to remain independent as long as possible (Australian Government 2013; Genet 2009; Scottish Government 2014). However, this means that, in many cases, people are being admitted into long-term care at a more advanced age than in previous years, in the most frail and dependent conditions, and with complex healthcare needs (Katz 2009). This is creating a shift within the sector from a previous focus on social care to one where older people living in care homes are likely to suffer from multiple morbidities, often over an extended period (Bowman 2014).

Unlike acute settings, it is rare for long-term care facilities to be staffed by well-qualified interdisciplinary clinical personnel, and access to external healthcare provision is often limited. In twothirds of the countries which responded to a recent World Health Organization/International Association of Gerontology and Geriatrics global survey, physicians are rarely involved in medical direction, and in one-sixth of the respondent countries they are never involved (Tolson 2013). In the United Kindgom (UK), although there are almost three times as many beds in long-term care as within the National Health Service (NHS) (Laing 2013), only $39 \%$ of these beds are in facilities with qualified nurses on site (Froggatt 2009). Staff retention is particularly challenging,

The champion for improved delivery of care to older people in long-term care settings: effects on professional practice, quality of care and resident outcomes (Protocol)

Copyright $\Subset 2015$ The Cochrane Collaboration. Published by John Wiley \& Sons, Ltd. 
as working within this sector is afforded low status compared to the higher-valued health sector (Tolson 2011) and there is a lack of well-developed career progression pathways (Cavendish 2013). The impact of staffing models on quality of care has been examined in a number of reviews which focus on this sector (Hodgkinson 2011; Spilsbury 2011). Because of the concerns over extrapolating evidence from acute and clinical sectors to long-term care of older people, organisations such as the National Institute for Health and Care Excellence have recently begun to develop guidance and quality standards specifically for the social care sector (NICE 2013a) . Despite the increasing clinical skill requirements posed by the vulnerability of the care home population, long-term care of older people "remains a low-wage economy" (Scottish Government and COSLA 2014) and significant increases in education and training budgets, either within the mainly for-profit sector or from external clinical education providers, are unlikely in the short to medium term. Within the current funding climate, educating long-term care staff in the clinical areas of care relevant to this sector is not a feasible option (Lee 2009). In an attempt to address the need for quality improvement, other models of intervention have been trialled (Ouslander 2007), one of which is to designate an individual staff member to be a topic 'champion'.

\section{Description of the intervention}

In areas of care which often prove resistant to improvement (e.g. oral health (Bassim 2008), nutrition (Gaskill 2009), continence care (Ouslander 2007), delirium (Siddiqi 2008), and infection control (Damschroder 2009)) one model of intervention being increasingly adopted is that of the 'champion'.

Outside long-term care, the term 'champion' is often adopted to characterise a number of diverse categories of roles, groups or individuals whose goal is to effect positive change (Shaw 2012; Soo 2009) and often refers to influential and often charismatic individuals with high social and institutional status (Shetty 2013). Because of its positive connotations, it is now used in a wide range of settings (Clarkson 2009; Jenkins 2014; Temoka 2013) leading to a level of conceptual confusion. In long-term care, however, it is most commonly used to denote a care home staff member who has either been designated or who volunteers to take on an additional level of responsibility in a particular topic area. Although 'champion' is the commonly-used term for this role in the UK, others such as aide (Bassim 2008), organiser (de Visschere 2010), or coordinator (Pronych 2010) can and do reflect similar roles.

Studies suggest that having at least one on-site staff member (normally a care assistant, healthcare worker, nursing auxiliary or nurse) who has undertaken some form of supplementary training in a specific topic area will help improve the practice in that area, the quality of care and the health outcomes for the residents (Damschroder 2009, Gaskill 2009, Lee 2009, Nicol 2005, Shaw 2012, Siddiqi 2008, Wardh 2002, Wardh 2003). They are likely to be a key contact person within the care home for the topic area (e.g. the dental team). In their realist review, Goodman 2015 suggests that having a nominated champion on particular health topics among care home staff being allocated to work with external healthcare providers is an essential attribute to 'relational working'.

Having a nominated champion for a certain aspect of care may be part of a multi-factor intervention (e.g. training for other staff, on-going support from health provider organisation, liaison link with health teams (e.g. dieticians, dental professionals)) or be the single element of an intervention.

\section{How the intervention might work}

Evidence-based educational interventions which focus on treating or alleviating common health problems experienced by people in long-term care are necessary but often not sufficient to change staff behaviour (Coleman 2006; Ouslander 2007). Evaluations often show, at best, short-term gains (Boczko 2009; Ouslander 2007). Sustainable change, especially in areas which are perceived as low priority, often proves elusive (Simons 2000) or resource intensive (Nicol 2005).

Wardh and colleagues (Wardh 2002) suggest that designating one or more staff members within a long-term care facility as champions, and giving these individuals additional training and responsibility for a specific topic area, (in this case, oral health), will result in the specially-trained staff member gaining the competence necessary to manage topic-related issues, liaise with the primary care team and encourage appropriate referrals in addition to overseeing the practice of colleagues.

Financial and time pressures together with regulations requiring specific staffing ratios can discourage managers from releasing staff to undertake non-mandatory education. Having an internal, specially-trained staff member may lead to less disruptive scheduling of training delivery, and allow for improved monitoring of the daily activities of the care staff (MacEntee 2007). An Australian clinical trial looking at nutrition coordinators in long-term care (Gaskill 2009) also found that staff were more likely to attend in-service sessions than those sessions more formally arranged by external providers. Although the findings of this study (i.e. improvements in nutritional status) were not statistically significant, additional training, raised awareness, responsibility, contact and visits helped contribute to improvements.

\section{Why it is important to do this review}

Best-evidence guidance for providing care to older people in long-term care settings exists for dementia (NICE 2010; Scottish Government 2011), nutrition (Dietitians of Canada 2013), oral health (NHS Health Scotland 2013), and falls (NICE 2013b). However an evidence-based approach to the implementation of guidelines is often lacking, and in other settings studies have shown that in order to achieve sustained healthcare improvements, guid- 
ance recommendations have to be embedded into routine clinical practice (Grimshaw 2004). One way of achieving this is through the appointment of a champion in a particular field of care to enable/facilitate implementation of guidelines (de Visschere 2010; Siddiqi 2008). Evaluations have been carried out on both standalone (Bassim 2008; Wardh 2003) and multi-component programmes (Siddiqi 2008) but little synthesis of the evidence for the effectiveness of champions for improving staff practice, quality of care and resident health outcomes within long-term care settings has been carried out.

\section{O B J E C T I VES}

To assess the effectiveness of the champion on professional practice, quality of care and resident outcomes in long-term care for older people.

\section{METHODS}

\section{Criteria for considering studies for this review}

\section{Types of studies}

We will include randomised controlled trials (RCTs) and cluster RCTs as these are considered the gold-standard study design to assess the effectiveness of an intervention. However, due to the context in which the intervention may be implemented (over time, within long-term care settings and appointing existing staff as champions), randomisation may not be feasible. If there are not enough of these studies, we will include non-randomised trials (NRTs). We will include controlled before-after (CBA) studies that assess outcomes prior to and after implementation in the intervention group and in the control group (where no intervention takes place). We will include interrupted time series (ITS) studies where outcomes are assessed over time, prior to and after the implementation of the intervention, controlling for any underlying secular trends. We will include repeated measures studies (RMS) where outcomes are measured repeatedly on the same individuals over time, prior to and after the implementation of the intervention.

We will use the following criteria for inclusion of CBA studies: (i) duration of pre- and post-intervention period should be comparable, (ii) baseline characteristics of intervention and control groups should be similar, and (iii) there should be at least two control and two intervention sites.

We will use the following criteria for inclusion of ITS studies: (i) a clearly-defined point in time when the intervention occurred, and (ii) at least three data points before and after the intervention.

\section{Types of participants}

Participants in this review will be staff working in, and older people residing in, long-term care facilities designated as providing personal and/or nursing care for older people. We will include studies in which the majority of residents are aged 65 or over.

\section{Types of interventions}

We will consider all forms of interventions aimed at changing staff behaviour with regard to their practice, the quality of care and resident outcomes, which have as a component an identified champion (as described above). We will exclude studies where the designated champion (or other relevant terms) is not an internal staff member of the care facility, or where the role does not involve the described elements, i.e. supplementary training, assumes additional responsibility for a specific topic area, and/or acts as key contact person with external healthcare team.

We will also exclude any studies in which the intervention involves a 'champion' where the term is used to refer to an external, highlevel, educationally-influential opinion leader such as those described in Flodgren 2011. Although the same term may be used to refer to such individuals, they are likely to be physicians and clinicians with higher social status, their leadership emerges rather than being a designated responsibility and their influence is considered to be related to influential communication networks and, as such, the role is not comparable to the care home champion as defined in this protocol.

We will compare the intervention to the following comparator groups:

1. No intervention (standard care), or

2. Single intervention (no champion), or

3. Multifaceted intervention (no champion).

\section{Types of outcome measures}

\section{Primary outcomes}

1. Staff adherence to recommended practice or guidelines

2. Staff change of behaviour associated with practice/ guidelines (e.g. twice daily teeth/denture cleaning)

\section{Secondary outcomes}

1. Resident objective health outcomes, depending on the focus of the intervention (e.g. delirium levels, continence, tissue viability, nutritional status, oral health status, emergency hospital admissions)

2. Resident quality of life (validated tools only)

3. Resident adverse health events (e.g. other than health outcome of interest- as a result of intervention focusing on one area of health, other areas of health are given lower priority, such

The champion for improved delivery of care to older people in long-term care settings: effects on professional practice, quality of care 
as delirium, dehydration, tissue viability, continence, nutritional status, oral health)

4. Resident satisfaction with care

\section{Search methods for identification of studies}

The review authors will develop a search strategy in collaboration with the Cochrane Effective Practice and Organisation of Care (EPOC) Group's Trials Search Coordinator (TSC).

\section{Electronic searches}

The TSC will search the Cochrane Database of Systematic Reviews and the Database of Abstracts of Reviews of Effects (DARE) for related systematic reviews, and the following databases for primary studies:

- Cochrane EPOC Group Specialised Register, Reference

Manager

- Cochrane Central Register of Controlled Trials (CENTRAL) (The Cochrane Library), Wiley (Search date)

- NHS Economic Evaluation Database, HTA Database (The Cochrane Library), Wiley (Search date)

- MEDLINE and MEDLINE In-Process and other non-

indexed citations, OvidSP (1946 - present)

- EMBASE, OvidSP (1974 - present)

- PsycINFO, OVIDSP (1967 - present)

- CINAHL (Cumulative Index to Nursing and Allied Health Literature), EbscoHost (1982 - present)

- Dissertations and Theses, ProQuest (Search date)

- Index to Theses (Search date)

- Science Citation Index Expanded, ISI Web of Knowledge

(1945 - present)

- Conference Proceedings Citation Index - Science, ISI Web of Knowledge (1990)

- Health Management Information Consortium (HMIC),

NHS Evidence (1979 - March 2013)

- Social Care Online (http://www.scie-

socialcareonline.org.uk)

- World Health Organization - International Clinical Trials

Registry Platform (Search date)

- US National Institutes of Health (clinicaltrials.gov)

We will adapt and translate the MEDLINE search strategy in Appendix 1 for other databases using appropriate syntax and vocabulary for those databases. The strategy includes Medical Subject Headings and synonyms for guidelines and implementation. Results will be limited by two methodological filters: the Cochrane Highly Sensitive Search Strategy (sensitivity- and precision-maximising version, 2008 revision) to identify randomised trials (Lefebvre 2011), and an EPOC methodology filter to identify non-RCT designs. There will be no language restrictions and studies will be included regardless of publication status.

\section{Searching other resources}

We will search reference lists of all papers and relevant reviews identified.

We will contact authors of relevant papers regarding any further published or unpublished work.

We will also contact authors of other reviews in the field of effective professional practice regarding relevant studies of which they may be aware.

We will conduct a grey literature search to identify studies not indexed in the databases listed above.

\section{Data collection and analysis}

\section{Selection of studies}

One review author (SW) will download all titles and abstracts retrieved by electronic searching to the reference management system EndNote (EndNote 2014), and remove duplicates. The remaining titles and abstracts will be independently examined by two review authors (AS and SW). We will directly exclude those studies which clearly do not meet the inclusion criteria and obtain full-text copies of potentially relevant references. Two review authors (AS, SW) will independently apply the inclusion criteria. Disagreements will be resolved by discussion, and where necessary arbitration by a third author. Eligible studies will be documented. Studies that are considered potentially eligible after the abstract reviewing stage, but are after scrutiny subsequently excluded, will be documented in the 'Characteristics of excluded studies' table ( EPOC 2014a). We will produce a PRISMA flowchart to document the selection process (Moher 2009).

\section{Data extraction and management}

Two review authors (AS and SW) will independently extract details of study design, population, intervention, comparison group and outcome measures from each included study using a modified and piloted version of the 'Good practice data extraction form' (EPOC 2014b). Other information to be extracted includes: the setting, whether the intervention was a complex or multifaceted intervention, the theoretical model used to underpin training of the champion (i.e. type of behaviour targeted); how the champion was identified/appointed/nominated. Authors will be contacted by email in the first instance and by telephone to follow-up nonresponders to retrieve any missing data or to provide clarification. Any disagreements between the two review authors will be adjudicated by a third author (GF).

\section{Assessment of risk of bias in included studies}

Two review authors (SW and AS) will assess the risk of bias independently using the 'Suggested risk of bias criteria' for EPOC 
reviews (EPOC 2014b). We will refer any disagreements to a third party for adjudication (GF). We will follow the suggested 'Risk of bias criteria' for EPOC reviews for (i) studies with a separate control group: RCTs, NRCTs, CBA studies and (ii) ITS studies (EPOC 2014c).

For studies with a separate control group, we will use nine 'Risk of bias' criteria :

1. Adequate sequence generation;

2. Adequate allocation concealment;

3. Were baseline outcome measurements similar?

4. Were baseline characteristics similar?

5. Were incomplete outcome data adequately addressed?

6. Was knowledge of the allocated interventions adequately prevented during the study?

7. Was the study adequately protected against contamination?

8. Was the study free from selective outcome reporting?

9. Was the study free from other risks of bias?

For ITS studies we will use the following seven 'Risk of bias' criteria:

1. Was the intervention independent of other events?

2. Was the shape of the intervention effect pre-specified?

3. Was the intervention unlikely to affect/influence data collection?

4. Was knowledge of the allocated interventions adequately prevented during the study?

5. Were incomplete outcome data adequately addressed?

6. Was the study free from selective outcome reporting?

7. Was the study free from other risks of bias?

We will assign an overall assessment of the risk of bias (high, low, unclear) to each of the included studies using the approach suggested in Chapter 8.7 of the Cochrane Handbook for Systematic Reviews of Interventions (Higgins 2011). We will establish the relative importance of different domains with respect to the review question, and identify key domains on which to make our assessment. Studies with low risk of bias for all key domains or where it seems unlikely that bias has seriously affected the results will be considered to have a low risk of bias. Studies in which risk of bias in at least one key domain was unclear or judged to have some bias that could plausibly raise doubts about the conclusions will be considered to have an unclear risk of bias. Studies with a high risk of bias in at least one key domain or judged to have serious bias that decreases the certainty of the conclusions will be considered to have a high risk of bias. We will incorporate the results into a 'Risk of bias' summary table and graphs for RCT and CBA studies; and separately for ITS studies.

\section{Measures of treatment effect}

We will report dichotomous outcomes as a risk ratio (RR) (relative risk) and risk difference (RD) with 95\% confidence intervals (CIs). For continuous outcomes, we will calculate the mean difference (MD) with 95\% CIs when all studies use the same outcome mea- sure. We will use the standardised mean difference (SMD) with 95\% CIs when studies measure the same outcome but use different methods. For ITS studies, we will abstract the difference in slope and the difference in level pre- to post-intervention. The postversus pre-intervention difference (adjusted for trends) at specific time-points (e.g. 3 and 12 months) will be reported. If the differences are not available in the primary reports, we will attempt reanalysis using data from graphs or tables based on the EPOC-specific guidance for analysis of ITS when the original study analysed the data inappropriately (EPOC 2014d).

\section{Unit of analysis issues}

If clustering has not been accounted for in the original analyses, we will use the intra-cluster correlation coefficient (ICC) to adjust the standard errors (or CIs) for the clustering. If the ICC is not published, we plan to estimate it from other similar studies, if they exist (Higgins 2011). However, sensitivity analyses will then be carried out to investigate the robustness of conclusions. For studies with unit of analysis errors, if there is insufficient information or data are not available, CIs and P values will not be reported.

\section{Dealing with missing data}

We will contact authors of studies to obtain data not available in the publication. We will deal with drop out of participants by using ntention-to-treat (ITT) analysis. We will use methods outlined by (Higgins 2011, Section 7.7.3) to estimate missing statistics such as standard deviations or correlation coefficients (regression coefficients) from CIs, standard errors, $t$ values, $\mathrm{P}$ values, and $\mathrm{F}$ values. Otherwise we may use imputation methods, provided the number of statistics to be estimated is low compared to the total number of included studies. We will conduct sensitivity analyses in this case, testing changes in assumptions made.

\section{Assessment of heterogeneity}

We will use a random-effects model in any meta-analysis, as it is anticipated that in a complex intervention, the true effect may vary according to context. We will calculate the $\mathrm{I}^{2}$ to explore statistical heterogeneity $\left(\mathrm{I}^{2}>30 \%\right.$ indicates moderate heterogeneity). If there is substantial methodological, statistical or clinical heterogeneity between studies, we will not proceed with meta-analysis (EPOC 2014d). We anticipate that due to the probability that included studies will have different interventions, and different outcome measures, substantial heterogeneity will exist, and therefore a narrative summary may be more appropriate. We will conduct a qualitative assessment of heterogeneity of the populations, settings and interventions from the included studies table before combining data. 


\section{Assessment of reporting biases}

We will check the original protocols for studies (if they exist) to assess the level of reporting bias. We will assess the risk of publication bias based on the characteristics of included studies. If there is a sufficient number of studies, we will consider using funnel plots to assess the risk of publication bias.

\section{Data synthesis}

We will pool the results of studies if at least two studies are homogenous regarding the participants, interventions and outcomes. For dichotomous outcomes, we will use RR and RD. For continuous outcomes we will use MD or SMD. We will use a more conservative random-effects model if some heterogeneity is identified (I ${ }^{2}>30 \%$ indicates moderate heterogeneity). We will calculate the median effect size and the interquartile range (IQR) within each study that reports more than one primary outcome.

If it is not possible to pool the results across studies due to high heterogeneity and/or differences in intervention and outcome measures, we will describe the impact of the interventions on outcomes in tabular form and carry out a qualitative assessment of the effect of the studies (EPOC 2014e). We will produce a summary of findings table (one for each main comparison) using the GRADE approach to assess the level of certainty of the evidence for each outcome (Guyatt 2011). We intend to use the GRADE worksheets and/or GRADEpro software to aid in the production of the summary of findings tables (GRADEproGDT 2008).and the assessment of the quality of the body of evidence.

\section{Subgroup analysis and investigation of heterogeneity}

The effect of an intervention may vary across studies due to variation in (i) long-term care setting and type of resident, (ii) the intensity of training (if any) provided to the champion, (iii) the method used to appoint the champion, and (iv) the different mechanisms of change employed by the intervention. If there are sufficient studies with the same outcomes, we will investigate subgroups as described. These investigations will be observational and we will compare the magnitude of the effects in different subgroups rather than conduct statistical tests between subgroups. We may use meta-regression to explore heterogeneity when it does not make sense to calculate an average effect across settings; training intensities; champion selection methods, or mechanisms of change. We may use visual analysis of tables (including standardised measures of effect and key explanatory factors), bubble plots and/or box plots.

\section{Sensitivity analysis}

In order to determine how robust and consistent the results are, we will conduct sensitivity analyses, based upon study design (RCT versus other) or risk of bias in study (high, moderate, low).

\section{ACKNOW LEDGEMENTS}

The review authors gratefully acknowledge the support of Gerd Flodgren and the Cochrane EPOC Group's UK Satellite, which is supported by an NIHR Cochrane Programme Grant.

\section{R E F E R E N C E S}

\section{Additional references}

\section{Australian Government 2013}

Australian Government. Department of Social Services 20122013 Report on the Operation of the Aged Care Act 1997.

Canberra: Australian Government, 2013.

Bassim 2008

Bassim CW, Gibson G, Ward T, Paphides BM, Denucci DJ. Modification of the risk of mortality from pneumonia with oral hygiene care. Journal of the American Geriatrics Society 2008;56(9):1601-7.

Boczko 2009

Boczko F, McKeon S, Sturkie D. Long-term care and oral health knowledge. Journal of the American Medical Directors Association 2009;10(3):204-6.

Bowman 2014

Bowman C, Meyer J. Formative care: defining the purpose and clinical practice of care for the frail. Journal of the Royal Society of Medicine 2014;107(3):95-8.
Cavendish 2013

Cavendish C. The Cavendish Review: an independent review into healthcare assistants and support workers in the NHS and social care settings. https://www.gov.uk/ government/publications/review-of-healthcare-assistantsand-support-workers-in-nhs-and-social-care (accessed 16 September 2015). London: The Department of Health, 2013.

Clarkson 2009

Clarkson JE, Bonetti D. Why be an evidence-based dentistry champion?. The Journal of Evidence-Based Dental Practice 2009;9(3):145-50.

\section{Coleman 2006}

Coleman P, Watson NM. Oral care provided by certified nursing assistants in nursing homes. Journal of the American Geriatrics Society 2006;54(1):138-43.

Damschroder 2009

Damschroder LJ, Banaszak-Holl J, Kowalski CP, Forman J, Saint S, Krein SL. The role of the champion in infection

The champion for improved delivery of care to older people in long-term care settings: effects on professional practice, quality of care 
prevention: results from a multisite qualitative study. Quality \& Safety in Health Care 2009;18(6):434-40.

\section{de Visschere 2010}

De Visschere L, Schols J, Van Der Putten G, De Baat C, Vanobbergen J. Effect evaluation of a supervised versus nonsupervised implementation of an oral health care guideline in nursing homes: a cluster randomised controlled clinical trial. Gerodontology 2012;29(2):e96-e106.

\section{Dietitians of Canada 2013}

Dietitians of Canada. Best practices for nutrition, food service and dining in long-term care homes. http:// www.osnm.org/webfm_send/597 (accessed 16 September 2015). Ontario: Dietitians of Canada, 2013.

EndNote 2014 [Computer program]

Thomson Reuters. EndNote. Version Z7. Thomson Reuters, 2014.

\section{EPOC 2014a}

Effective Practice, Organisation of Care (EPOC). Characteristics of excluded studies. EPOC Resources for review authors. Oslo: Norwegian Knowledge Centre for the Health Services, 2014. Available at: http:// epocoslo.cochrane.org/epoc-specific-resources-reviewauthors.

\section{EPOC 2014b}

Effective Practice, Organisation of Care (EPOC). Good practice data extraction form. EPOC Resources for review authors. Oslo: Norwegian Knowledge Centre for the Health Services, 2014. Available at: http://epocoslo.cochrane.org/ epoc-specific-resources-review-authors.

\section{EPOC 2014c}

Effective Practice, Organisation of Care (EPOC). Suggested risk of bias criteria for EPOC reviews. EPOC Resources for review authors. Oslo: Norwegian Knowledge Centre for the Health Services, 2014. Available at: http:// epocoslo.cochrane.org/epoc-specific-resources-reviewauthors.

\section{EPOC 2014d}

Effective Practice, Organisation of Care (EPOC). Analysis in EPOC reviews. EPOC Resources for review authors. Oslo: Norwegian Knowledge Centre for the Health Services, 2014. Available at: http://epocoslo.cochrane.org/ epoc-specific-resources-review-authors.

\section{EPOC 2014e}

Effective Practice, Organisation of Care (EPOC). Synthesising results when it does not make sense to do a meta-analysis. EPOC Resources for review authors. Oslo: Norwegian Knowledge Centre for the Health Services, 2014. Available at: http://epocoslo.cochrane.org/ epoc-specific-resources-review-authors.

\section{Flodgren 2011}

Flodgren G, Parmelli E, Doumit G, Gattellari M, O’Brien MA, Grimshaw J, et al. Local opinion leaders: effects on professional practice and health care outcomes. Cochrane Database of Systematic Reviews 2011, Issue 8. [DOI: 10.1002/14651858.CD000125.pub4]

\section{Froggatt 2009}

Froggatt K, Davies S, Meyer J. Understanding Care Homes. London: Jessica Kingsley Publishers, 2009.

\section{Gaskill 2009}

Gaskill D, Isenring EA, Black LJ, Hassall S, Bauer JD. Maintaining nutrition in aged care residents with a trainthe-trainer intervention and Nutrition Coordinator. Journal of Nutrition, Health \& Aging 2009;13(10):913-7.

\section{Genet 2009}

Genet G, Boerma WGW, Kringos DS, Bouman A, Francke AL, Fagerstrom C, et al. Home care in Europe: a systematic literature review. BMC Health Services Research 2011;11: 207-14. [DOI: 10.1186/1472-6963-11-207]

\section{Goodman 2015}

Goodman C, Davies SL, Gordon AL, Meyer J, Dening T, Gladman JRF, et al. Relationships, expertise, incentives, and governance: supporting care home residents' access to health care. An interview study from England. Journal of the American Medical Directors Association 2015;16(5):427-32.

GRADEproGDT 2008 [Computer program] Brozek J, Oxman A, Schünemann H. GRADEprofiler. 3.2 for Windows. GRADE Working Group, 2008.

\section{Grimshaw 2004}

Grimshaw JM, Thomas RE, MacLennan G, Fraser C, Ramsay CF, Vale L, et al. Effectiveness and efficiency of guideline dissemination and implementation strategies. Health Technology Assessment 2004;8(16):iii-iv, 1-72.

\section{Guyatt 2011}

Guyatt GH, Oxman AD, Schünemann HJ, Tugwell P, Knottnerus A. GRADE guidelines: a new series of articles in the Journal of Clinical Epidemiology. Journal of Clinical Epidemiology 2011;64(4):380-2.

\section{Higgins 2011}

Higgins JPT, Green S (editors). Cochrane Handbook for Systematic Reviews of Interventions Version 5.1.0 [updated March 2011]. The Cochrane Collaboration, 2011. Available from www.cochrane-handbook.org.

\section{Hodgkinson 2011}

Hodgkinson B, Haesler EJ, Nay R, O’Donnell MH, McAuliffe LP. Effectiveness of staffing models in residential, subacute, extended aged care settings on patient and staff outcomes. Cochrane Database of Systematic Reviews 2011, Issue 6. [DOI: 10.1002/14651858.CD006563.pub2]

\section{Jenkins 2014}

Jenkins J, Calzone KA. Genomics nursing Faculty Champion initiative. Nurse Educator 2014 Jan-Feb;39(1): 8-13.

Katz 2009

Katz PR, Karuza J, Intrator O, Mor V. Nursing home physician specialists: a response to the workforce crisis in long-term care. Annals of Internal Medicine 2009;150(6): 411-3. 
Laing 2013

Laing, Buisson. Care of Elderly People UK Market Survey

2012/13. www.laingbuisson.co.uk. London: Laing \& Buisson, 2013.

Lee 2009

Lee R, Scott F. Competent to care. A train-the-trainer method of teaching as a way of implementing the correct use of the 'Malnutrition Universal Screening Tool' in Norfolk: is it effective?. Proceedings of the Nutrition Society 2009;68 (3):300-5.

\section{Lefebvre 2011}

Lefebvre C, Manheimer E, Glanville J. Chapter 6: Searching for studies. In: Higgins JPT, Green S editor(s). Cochrane Handbook for Systematic Reviews of Interventions Version 5.1.0 (updated March 2011). The Cochrane Collaboration, 2011. Available from: www.cochrane-handbook.org..

\section{MacEntee 2007}

MacEntee MI. Quality of life as an indicator of oral health in older people. Journal of the American Dental Association 2007;138(Supplement):47S-52S.

\section{Moher 2009}

Moher D, Liberati A, Tetzlaff J, Altman DG for the PRISMA Group. Preferred reporting items for systematic reviews and meta-analyses: the PRISMA statement.. BMJ 2009;339:b2535-b2535.

NHS Health Scotland 2013

NHS Health Scotland. Caring for smiles - Guide for care homes: better oral health for dependent older people. http: //www.nes.scot.nhs.uk/media/2603965/caring_for_smiles_ guide_for_care_homes.pdf (accessed 16 September 2015). Edinburgh: NHS Health Scotland, 2013.

\section{NICE 2010}

National Institute for Health and Care Excellence. Dementia Quality Standard. https://www.nice.org.uk/ guidance/QS1 (accessed 15 September 2015). London: National Institute for Health and Care Excellence, 2010.

\section{NICE 2013a}

National Institute of Care and Health Excellence. NICE and Social Care. http://www.nice.org.uk/About/NICECommunities/Social-care (accessed 10 June 2015) 2013.

\section{NICE 2013b}

National Institute for Health and Care Excellence. Falls: assessment and prevention of falls in older people, NICE Guidelines CG161. http://www.nice.org.uk/guidance/ cg161 (accessed 16 September 2015). London: National Institute for Health and Care Excellence, 2013.

Nicol 2005

Nicol R, Sweeney MP, McHugh S, Bagg J. Effectiveness of health care worker training on the oral health of elderly residents of nursing homes. Community Dentistry and Oral Epidemiology 2005;33(2):115-24.

Ouslander 2007

Ouslander JG. Quality improvement initiatives for urinary incontinence in nursing homes. Journal of the American
Medical Directors Association 2007;8(3 Supplement):

S6-S11.

\section{Pronych 2010}

Pronych GJ, Brown EJ, Horsch K, Mercer K. Oral health coordinators in long-term care - a pilot study. Special Care Dentistry 2010;30(2):59-65.

Scottish Government 2011

Scottish Government. Promoting excellence: a framework for all health and social services staff working with people with dementia, their families and carers. http:// www.gov.scot/Publications/2011/05/31085332/0 (accessed 16 September 2015). Edinburgh: APS Group Scotland, 2011.

\section{Scottish Government 2014}

Scottish Government. Draft National Health and Wellbeing Outcomes. http://www.scotland.gov.uk/Topics/Health/ Policy/Adult-Health-SocialCare-Integration/Outcomes (accessed 16 September 2015) 2014.

\section{Scottish Government and COSLA 2014}

Scottish Government and COSLA: Task force for the future of residential care in Scotland. Recommendations for the future of residential care for older people in Scotland, Summary. http://www.gov.scot/Resource/0044/ 00444594.pdf (accessed 16 September 2015). Edinburgh: Scottish Government, 2014.

Shaw 2012

Shaw EK, Howard J, West DR, Crabtree BF, Nease DE, Tutt B, et al. The role of the champion in primary care change efforts: from the State Networks of Colorado Ambulatory Practices and Partners (SNOCAP). Journal of the American Board of Family Medicine: JABFM 2012 Sep-Oct;25(5):676-85.

Shetty 2013

Shetty P. Martin McKee: champion of public health in Europe. Lancet 2013 Mar 30;381(9872):1089.

\section{Siddiqi 2008}

Siddiqi N, Young J, Cheater FM, Harding RA. Educating staff working in long-term care about delirium: the Trojan horse for improving quality of care?. Journal of Psychosomatic Research 2008;65(3):261-6.

\section{Simons 2000}

Simons D, Baker P, Jones B, Kidd EA, Beighton D. An evaluation of an oral health training programme for carers of the elderly in residential homes. British Dental Journal 2000;188(4):206-10.

Soo 2009

Soo S, Berta W, Baker GR. Role of champions in the implementation of patient safety practice change. Healthcare Quarterly 2009;12(Spec No Patient):123-8.

\section{Spilsbury 2011}

Spilsbury K, Hewitt C, Stirk L, Bowman C. The relationship between nurse staffing and quality of care in nursing homes: a systematic review. International Journal of Nursing Studies 2011;48(6):732-50.

The champion for improved delivery of care to older people in long-term care settings: effects on professional practice, quality of care 
Temoka 2013

Temoka E. Becoming a vaccine champion: evidence-based interventions to address the challenges of vaccination. South Dakota Medicine: The Journal of the South Dakota State Medical Association 2013;(Spec no.):68-72.

\section{Tolson 2011}

Tolson D. Promoting evidence informed improvements in care homes: nursing perspectives. Journal of Nutrition Health and Ageing 2011;15(5):420-2.

\section{Tolson 2013}

Tolson D, Rolland Y, Katz PR, Morley JE, Vellas B. An international survey of nursing homes. Journal of the American Medical Directors Association 2013;14(7):459-62.

\section{Wardh 2002}

Wardh I, Berggren U, Hallberg, LR, Andersson L, Sorensen

$S$. Dental auscultation for nursing personnel as a model of oral health care education: development, baseline, and 6-month follow-up assessments. Acta Odontologica Scandinavica 2002;60(1):13-9.

\section{Wardh 2003}

Wardh I, Hallberg LR, Berggren U, Andersson L, Sorensen $S$. Oral health education for nursing personnel; experiences among specially trained oral care aides: one-year followup interviews with oral care aides at a nursing facility. Scandinavian Journal of Caring Sciences 2003;17(3):250-6.

* Indicates the major publication for the study

\section{A P P E N D I C E S}

\section{Appendix I. MEDLINE search strategy}

1. (elder $\$$ or geriatric or geriatrics or old or older or aged or senior or seniors or veteran\$).mp. [mp=title, abstract, original title, name of substance word, subject heading word, keyword heading word, protocol supplementary concept, rare disease supplementary concept, unique identifier]

2. (champion or champions or coordinator\$ or Facilitator\$ or aide or aides or carer or "care staff" or "healthcare worker" or "certified nursing assistant" or ausculation).mp. [mp=title, abstract, original title, name of substance word, subject heading word, keyword heading word, protocol supplementary concept, rare disease supplementary concept, unique identifier] 
4. ("long term" or "long-term" or "care home\$" or "nursing home\$" or "care of the elderly" or "continuing care" or "sub acute" or "subacute" or "sub-acute" or "residential care").mp. [mp=title, abstract, original title, name of substance word, subject heading word, keyword heading word, protocol supplementary concept, rare disease supplementary concept, unique identifier]

5. 1 and 2 and 4

6. ("long term" or "long-term" or "care home\$" or "nursing home\$" or "continuing care" or "sub acute" or "subacute" or "sub-acute" or "residential care").mp. [mp=title, abstract, original title, name of substance word, subject heading word, keyword heading word, protocol supplementary concept, rare disease supplementary concept, unique identifier]

7. 1 and 2 and 6

8. (champion or champions or coordinator\$ or facilitator or aide or aides or "train the trainer").mp. [mp=title, abstract, original title, name of substance word, subject heading word, keyword heading word, protocol supplementary concept, rare disease supplementary concept, unique identifier]

9. $\quad 1$ and 6 and 8 
10. (champion or champions or coordinator $\$$ or facilitator or aide or aides).mp. [mp=title, abstract, original title, name of substance word, subject heading word, keyword heading word, protocol supplementary concept, rare disease supplementary concept, unique identifier]

11. 1 and 6 and 10

12. 1 and 10

\section{CONTRIBUTIONSOFAUTHORS}

AS and SW conceived the review, drafted the protocol and developed the search strategy. GF reviewed the protocol.

\section{DECLARATIONSOF INTEREST}

None.

\section{SOURCES OF SUPPORT}

\section{Internal sources}

- The University of Glasgow Dental School, UK. Salary

- NHS Ayrshire \& Arran, UK.

Salary

\section{External sources}

- No sources of support supplied 